\title{
Harmonizations of time with non periodic ordered structures in discrete geometry and astronomy
}

\author{
Juan García Escudero \\ Facultad de Ciencias, Universidad de Oviedo \\ jjge@ pinon.ccu.uniovi.es
}

\begin{abstract}
Non periodic ordered tilings can be used for the generation of discrete structures in the time and frequency domains. The Fourier spectrum of certain aperiodic ordered temporal sequences described by Lindenmayer systems shows a discrete part. In order to appy these ideas the main tools belong to discrete geometry and number theory. These techniques provide a connection between rhythms and harmonic fields which may have a natural phenomena basis when observational data of certain types of variable stars are analyzed. The pulsation of some semiregular and delta scuti stars is reflected in their light curves which can be modelled by means of sinusoidal sequences related with the golden number. ${ }^{1}$
\end{abstract}

\section{Introduction}

In recent works I have explored relations between aperiodic but ordered temporal sequences, harmonic fields arising from the Fourier analysis of such sequences and of instrumental sounds, and sounds synthesized starting from the dynamic spectra appearing in the analysis. Time is structured with 1D geometric sequences generating aperiodic ordered rhythms. The use of number theory shows that some of them are transparent in the sense that their Fourier spectra have a discrete component and provide the pairs intensity-pitch. The resulting harmony is an extension of the classical harmony which is based on the spectra of periodic rhythms. Due to the lack of translational symmetry, the temporal spectra are always inharmonic. The central frequencies in additive synthesis of filtered noises have been obtained from the basic temporal and instrumental spectra. Their amplitudes evolve according to successive iterations previous to the amplitudes stabilization. The bandwidths in (Escudero 1997) have been introduced according to perceptual criteria, but 1D non periodic ordered tilings can show also continuous components in their Fourier

\footnotetext{
${ }^{1}$ An extended version of this paper will be submitted to the Springer Lecture Notes in Computer Science series
}

transform . In other works spectra of instrumental or vocal sounds are part of autonomous harmonic fields or in interaction with those corresponding to the spectra arising in the temporal structures. The scaling factors of the aperiodic ordered 1D sequences are algebraic integers : roots of monic (the leading coefficient is equal to 1 ) polynomials with integer coefficients. Certain connections with observational data in astronomy may also be established and the time can be structured in a natural phenomena basis.

\section{Fourier spectra of non periodic or- dered temporal sequences}

An approach to continuity can be obtained in computer music compositions due to the high accuracy of the medium. It has been also the basis of instrumental works by authors like Brian Ferneyhough: the pitches, dynamics and rhythms are chosen from a parametric space as continuous as possible from a perceptual point of view. Local realizations are, except in some cases like glissandi or dynamic changes, essentialy discrete. Several authors have looked in the past for discrete time structures that would be related to organizations of pitch structures (Cowell 1996). By decreasing the duration of a series of impulses periodicaly distributed in time, Stockhausen pointed out how the same basic process is behind our perception of duration and pitch (Stockhausen 1963) . Longer durations define temporal scales related with the form and its articulations.

A possible way to structure the time is by means of non periodic ordered sequences which can be described algebraically in terms of Lindenmayer systems. A OL-system (Rozenberg and Salomaa 1980) is a triple $G=\{\Sigma, r, \omega\}$ where $\Sigma$ is an alphabet, $r$ is a finite substitution on $\Sigma$ into the set of subsets of $\Sigma^{*}$, and $\omega$ is the axiom. G is called a DOL-system if $\#(r(x))=1$, for every $x \in \Sigma$.

Consider the alphabet $\Sigma=\{a, b\}$, the production rules:

$$
a \longmapsto a b a a, \quad b \longmapsto a a b
$$


and the axiom $a$. The language consists in the words $a, a b a a$, abaaaababaaabaa,... If we associate to $a$ and $b$ temporal segments (rhythmic units) of lengths $l_{a}, l_{b}$ with $l_{a} / l_{b}=(\alpha-$ 1) $/ 2$, where $\alpha$ is the highest root of the polynomial $x^{2}-$ $4 x+1$, we get a selfsimilar sequence with scaling factor $\alpha$. Although $\alpha$ is not rational, rational approximants can be obtained for the basic rhythmic units by using the recursion relations

$$
a_{n+1}=3 a_{n}+b_{n}, \quad b_{n+1}=2 a_{n}+b_{n}
$$

The frequencies forming the discrete part of the Fourier transform of impulses distributions following the sequence (1) have the form :

$$
f=p \nu_{1}+q \nu_{2}
$$

where $p$ and $q$ are arbitrary integers and $\nu_{1}=(\alpha-1) /(\alpha+1)$, $\nu_{2}=2 /(\alpha+1)$. The duration $t_{n}$ of the temporal sequence after iterating $n$ times the production rules to the axiom $a$ satisfies

$$
t_{n+1}=4 t_{n}-t_{n-1}
$$

and initial conditions $t_{0}=l_{a}, t_{1}=3 l_{a}+l_{b}$. The recursion relations for the Fourier amplitudes are

$$
\begin{aligned}
& F_{n+1}= \\
& F_{n}\left(1+\exp \left(i f t_{n}\right)+\exp \left(2 i f t_{n}\right)+\exp \left(i f\left(3 t_{n}-t_{n-1}\right)\right)\right)- \\
& F_{n-1} \exp \left(i f\left(3 t_{n}-t_{n-1}\right)\right)
\end{aligned}
$$

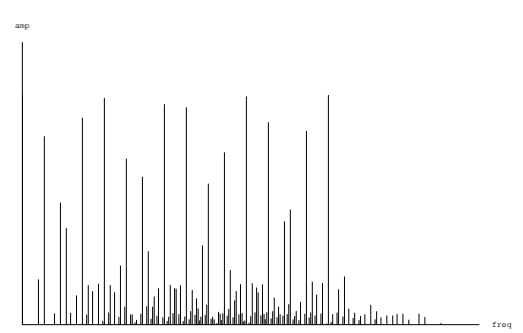

Fig.1. Plot of the normalized Fourier amplitudes for the absystem .

Four iterations are needed in order to stabilize the quotient between the amplitudes and the number of impulses (Fig.1). Observe that no units have been specified because they depend on the context (harmonic fields or sound synthesis) where they appear. Although the spectrum is a dense set, above a certain amplitude thereshold the number of frequencies which are lower than a given one is finite. Static spectra with pitches separated in the frequency space by distances of two lengths can be obtained. The first pitches of a spectrum with this property are:

$\{1,0,58, f,-0.95\},\{2,0,115, p p,-1.7\},\{2,1,158, f f, 0.7\}$, $\{3,1,215, f f,-0.3\},\{4,1,273, m f,-1.2\}$,

$\{4,2,315, m f, 1.3\},\{5,2,373, f f, 0.4\},\{6,2,431, f f,-0.5\}$, where in each quadruple we use the notation:

$\{p, q$, frequency $(H z)$, intensity, phase $\}$, and $p, q$ are the integers in eq.3.

In (Escudero 2001) several planar tilings with twelve-fold symmetry have been generated. A biological motivation is behind tiling growth: many cell divisions in multicellular organisms occur at the same time. The recursive structure of the figures is captured in terms of word sequences in bracketed Lindenmayer systems where productions are applied in parallel and simultaneously replace all the letters in a given word. This type of word sequences, which were introduced for the description of Penrose patterns (Escudero 1995), have been used in the formal constructions of (Escudero 1993). The prototiles may have also a correspondence in terms of elementary harmonic fields. They can be derived from the prototile edges or the spectra (local periodicities can give chords close to those classified by the classical harmony ) and the words describing the tiling growth produce a hierarchy of chord sequences. The prototile edges in (Escudero 2001) are $A=s_{1}, B=s_{2}, C=s_{3}, D=s_{4}, E=s_{5}, F=s_{6}$. where $s_{l}=\sin (l \pi / 12)$. An arrow is placed on all the edges except $F$. For a given prototile their edges are labelled with 0,1 depending on whether the arrow orientation with respect to the prototile interior is anticlockwise or clockwise respectively. The substitution rules $X \longmapsto \phi[X]$ are :

$$
\begin{array}{llrl}
\phi\left[A^{0}\right] & =E^{1} & & \phi\left[B^{0}\right]=D^{0} F \\
\phi\left[C^{0}\right] & =E^{1} E^{0} C^{1} & & \phi\left[D^{0}\right]=D^{0} F D^{1} B^{0} \\
\phi\left[E^{0}\right] & =A^{1} C^{0} E^{1} E^{0} C^{1} & & \phi[F]=B^{1} D^{0} F D^{1} B^{0}
\end{array}
$$

and $\phi\left(L^{i}\right)=\operatorname{Mir}\left(t\left(\phi\left(L^{i+1}\right)\right)\right)$ where $M i r$ denotes the mirror reflection of a word $i \in Z_{2}$ and the map $t$ increases in one unit the index.

In what follows we use the rhythmic notation of Patchwork-

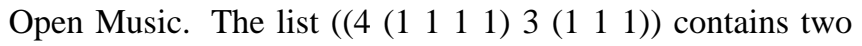
sub-lists each one representing a bar, the number before each sub-list denotes the number of pulsations and negative numbers indicate rests. For instance 4( -211 ) denotes a half-note rest followed by two quarter-notes. In Yod, a work for six percussionists and computer, the prototile edges substitution rules given in eq.6 play a major role. It is possible to define also rational approximants for the temporal segments $s_{l}$. The 
last section of Yod begins with the following rhythmic pattern in the first bar:

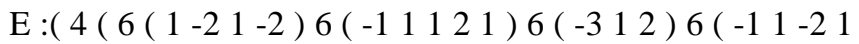

$-1))$

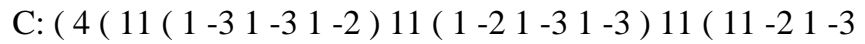

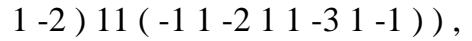

$\mathrm{F}:\left(\begin{array}{l}4 \\ (11\end{array}\right.$

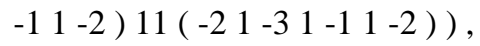

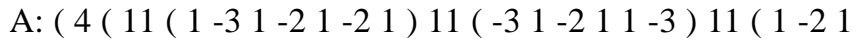

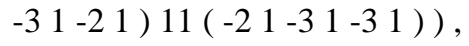

$\mathrm{D}:\left(\begin{array}{c}4 \\ (\end{array}\right.$

$\left.\begin{array}{lllll}-1 & 1 & -2 & 1 & -2\end{array}\right) 16\left(\begin{array}{llllllllllllllllllll}-1 & 1 & -2 & 1 & -3 & 1 & -2 & 1 & -1 & 1 & -1 & 1\end{array}\right) \begin{array}{llll}-2 & 1 & -2 & 1\end{array}$

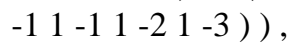

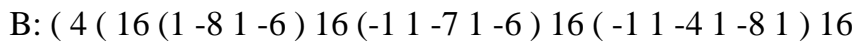

( -7 1 $1-8))$.

The letter preceding each sequence is the axiom that generates it. Observe that there are two independent subsets $\{A, E, C\},\{B, D, F\}$ in eq.6 which transform into themselves and therefore every sequence has only three rhythmic units. They are constructed in such a way that the only whole coincidences are at the begining and at the end of the section.

\section{Pulsations of variable stars and sub- stitutional sequences}

In addition to the aperiodic ordered structures which have a mathematical motivation, spectra of natural phenomena in astronomy can be used as part of the pitch and rhythmic organizations. Stellar pulsations may be irregular, semi-regular or periodic. In this work we consider stars with light curve variations related with the structures previously discussed. Semiregular stars like UW Herculis and some Delta Scuti stars have a multimode pulsation with a light curve that can be modelled by concatenation of sinusoidal fragments following word sequences in formal grammars. The best known example of a selfsimilar quasiperiodic sequence is the Fibonacci sequence. It is related with the ubiquitous golden number . Lindenmayer systems can be used in order to describe the Fibonacci sequences. The alphabet is $\{L, S\}$, the production rules $r:\{L \longmapsto L S, S \longmapsto L\}$ and the axiom $L$. The sequence consists in the words $L, L S, L S L, L S L L S, \ldots$. A $1 \mathrm{D}$ quasiperiodic geometric structure can be obtained if $L$ and $S$ represent two segments with a ratio equals the golden number $\tau$. The sequence is deterministic in the sense that only one word is allowed with a given length. The Fibonacci numbers $F(n)$ can be defined with the help of the recurrence relation $F(n+2)=F(n+1)+F(n), F(0)=F(1)=1$. By iterating this relation we get the sequence $1,1,2,3,5,8,13,21 \ldots$ The quotient of two succesive Fibonacci numbers approaches the golden number when $n$ increases. The light curve of the Delta Scuti star V346 Orionis can be modelled with a curve constructed by concatenation of two sinusoidal fragments with two lengths in a golden ratio. For the observations taken on the night of 4 November 2001 at the 1.52 telescope in Loiano (Italy) (Pinheiro et al,2003), the temporal segments have been chosen by matching with the empirical dataset. The axiom is $S L$ and the derivation corresponds to $r^{3}(S L)=L S L L S L L S$. The rhythmic structure corresponding to the rational approximants $L=3, S=2$ is

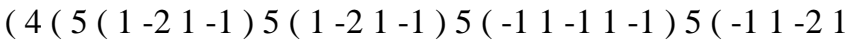
)$)$

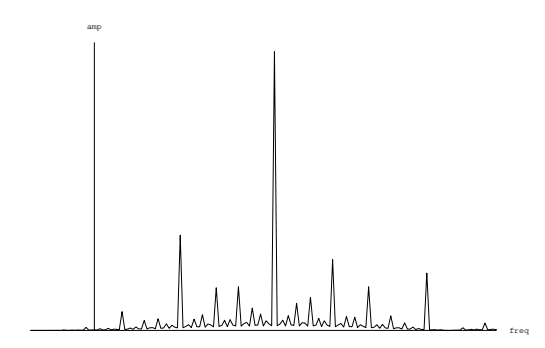

Fig.2. Normalized Fourier amplitudes for the curve with sinusoidal fragments following a Fibonacci sequence with 144 symbols.

The Fourier spectrum of an artificial light curve with two sinusoidal fragments with lengths $L=F(10)=89$ and $S=F(9)=55$ units following a Fibonacci sequence can be seen in Fig. 2 The analysis corresponds to the word $r^{11}$ with 144 letters (compare (Escudero 2003) for the analysis corresponding to the word with 89 letters ). The periods corresponding to the peaks with highest amplitudes in Fig. 2 have 123 and 76 units, a factor of $3-\tau$ in relation with the durations of the sinusoidal fragments. The presence of sharp peaks is due to the fact that the golden number, like $\alpha$ is a Pisot-Vijayaraghavan number, namely an algebraic integer with all its conjugates (the remaining roots of its minimal polynomial, in this case $x^{2}-x-1$ ) strictily less than one in absolute value (Bombieri and Taylor 1986). In (Hollander and Solomyak 2003) it is shown that any Pisot substitution dynamical system on two symbols has pure discrete spectrum. The frequencies of the Fourier spectrum of Fibonacci sequences belong to a dense set which consists of the linear combinations with integer coefficients of two fundamental frequencies: $f=m_{1} \omega_{1}+m_{2} \omega_{2}$, where $\omega_{1}$ and $\omega_{2}$ are in a golden ratio and $m_{1}, m_{2}$ are integers. The spectrum stabilizes 
when the iteration level is increased. The sharp peaks appear in the analysis when the word length is long enough. For shorter words the peaks appear with a certain bandwidth and this fact can be used in order to get dynamic spectra for direct synthesis of sound by appropriate scaling the basic units.

Other stars like the semiregular UW Herculis can be studied with the help of models based on non periodic ordered sinusoidal sequences. A stochastic 0L-system is a 4-tuple $G=$ $\{\Sigma, P, \omega, \pi\}$ where $P$ is a set of productions $r_{i}$ and $\pi: P \longmapsto$ $(0,1]$ is a probability distribution. We define a Fibonacci stochastic L-system with $P=\left\{r_{1}, r_{2}\right\}$ and $r_{1}:\{L \longmapsto$ $L S, S \longmapsto L\}, r_{2}:\{L \longmapsto S L, S \longmapsto L\}$. The Fourier spectrum of artificial data generated by following the word sequences of this system contains peaks with bandwidths. When the amplitudes are increased at certain positions according with the observations some of the pitches of the spectrum with significant amplitudes are (only the frequency in $\mathrm{Hz}$ and the intensity are specified in each pair)

$\{53, f\},\{124, m f\},\{178, f\},\{231, f\},\{267, m f\}$, $\{320, m f\},\{373, f\},\{427, m f\},\{498, m f\},\{551, m f\}$, $\{605, f f\},\{960, m p\}$,

which corresponds to a certain period of time. Dynamic spectra can be generated by considering different periods of time

\section{Concluding remarks}

In this paper specific techniques with a basis in number theory, geometry and astronomy have been discussed in an attempt to implement, in a different context, formalized mechanisms founded on the mathematics of aperiodic ordered systems. A common intention is the computation of static and dynamic spectra of temporal sequences having a mathematical or physical motivation. The combination of spectra computed from temporal sequences with other techniques like granular synthesis (Roads 2001) can be a powerful tool for experimentation in sound design. The temporal sequences and their spectra have been used as part of the structural basis of several works like Invertida Raíz for nine instrumentalists and computer, where the timbric integrations between the electronic and instrumental parts are produced with hybrid synthesis techniques, or Ad Matutinum for mixed choir where the harmonic fields are generated from the spectra of both vowels and temporal structures. The deterministic sequences are selfsimilar although,when they are included in a compositional context, this property is usually distorted at some level in the hierarchy . Grammars describing the generation of non periodic tilings have been used in the formal constructions since (Escudero 1993). Other interesting results can be obtained by using stochastic sequences (Escudero 2000). The analysis of aperiodic ordered sequences can provide a unified basis from the point of view of the formalization process although without any type of a priori aesthetic consistency in the final results. The relationship at a perceptual level between the generated aperiodic ordered rhythms and their associated spectra must be explored in a deeper level in order to expand our tools for algorithmic composition.

\section{References}

Bombieri,E. and Taylor,J.E. 1986. "Which Distributions of Matter Diffract ?. An initial investigation", Journal de Physique France, C3,Vol.47,19-28.

Cowell H. 1996. New Music Resources. Cambridge University Press.

Escudero,J.G.1993. La Tierra Ubérrima for string quartet. CD Agenda Edizioni Musicali 2003-020

Escudero,J.G.1995 Grammars for Icosahedral Danzer Tilings. Journal of Physics A: Mathematical and General. Vol.28, 5207-5215.

Escudero,J.G.1997. Estudio del Tiempo Iluminado II for computer. CD MasterVision 001. Concorso Internazionale di Composizione Elettronica "Pierre Schaeffer". SIAE.

Escudero,J.G.1999. Time and Frequency Structures within Selfsimilar Aperiodic Geometries. In H.G.Feichtinger and M.Doerfler (eds). Diderot Forum on Mathematics and Music. Oesterreichische Computer Gesellschaft Series. Viena,Vol.133, 145-152

Escudero,J.G.2000. Continuous and Discrete Fourier Spectra of Aperiodic Sequences for Sound Modeling. In D.Rocchesso and M.Signoretto (eds.) Proceedings Digital Audio Effects Workshop DAFX00 .Universitá degli Studi di Verona, .265268

Escudero,J.G.2001 ET0L-Systems for Composite Dodecagonal Quasicrystal Patterns. International Journal of Modern Physics B. Vol.15,1165-1175.

Escudero,J.G. 2003. Fibonacci Sequences and the Multiperiodicity of the Variable Star UW Herculis.Chinese Journal of Astronomy and Astrophysics. Vol.3, 235-240

Hollander M and Solomyak B.2003. Two-symbol Pisot substitutions have pure discrete spectrum. Ergodic Theory and Dynamical Systems. Vol.23.533-540.

Pinheiro, F et al. 2003. Oscillations in the PMS Delta Scuti star V346 Ori. Astronomy and Astrophysics.Vol.399,271274.

Roads, C. 2001. Microsound. The MIT Press. Cambridge. Massachusetts.

Rozenberg G. and Salomaa A.1980. The Mathematical Theory of L Systems. Academic Press. New York.1980.

Stockhausen K,, 1963 ...wie die Zeit vergeht... Texte zur elektronischen und instrumentalen Musik. Bd.1. Dumont Buchverlag, Kln. 